\title{
OPTIMISMO, AFRONTAMIENTO Y CÁNCER PEDIÁTRICO: UN ESTUDIO DESCRIPTIVO
}

\author{
OPTIMISM, COPING AND CHILDHOOD CANCER: A DESCRIPTIVE STUDY
}

Carmina Castellano-Tejedor

Departmento de Psiquiatría, Hospital Universitari Vall d'Hebron, CIBERSAM, Universidad Autónoma de Barcelona, España.

Departmento de Psicología Básica, Universidad Autónoma de Barcelona, Barcelona, España.

Resumen

Objetivo y métodos: El presente trabajo tiene por objetivo evaluar los niveles de optimismo en los peores momentos del proceso oncológico (auto-referidos) en una muestra de adolescentes oncológicos en remisión $(\mathrm{N}=41)$. Asimismo, se explorará si dicho optimismo se asocia con el afrontamiento llevado a cabo en esos momentos $y$; por otra parte, con la calidad de vida (CVRS) resultante en periodo de supervivencia.

Resultados: Un $\mathbf{7 0 , 7 \%}$ de adolescentes se manifestaron optimistas en los peores momentos de su enfermedad. Los adolescentes considerados "pesimistas" mostraron hacer un mayor uso de las estrategias de "auto-inculparse" y "buscar apoyo espiritual" que los "optimistas". Por último, sólo se observó una relación significativa entre el optimismo durante los momentos más difíciles del proceso oncológico y la dimensión de "bienestar físico" de la CVRS resultante en periodo de remisión.

Conclusiones: No ha podido observarse un efecto claro del optimismo durante el cáncer y la CVRS resultante. Así, podría ser que el optimismo fuese una condición necesaria para sobrellevar la enfermedad de un modo más adaptativo, pero quizás no es suficiente como para suponer una mayor CVRS una vez el cáncer ha remitido. Incluso es probable que el optimismo pueda facilitar la ocurrencia de otro
Abstract

Purpose and methods: This study is aimed to assess optimism in the worst self-reported moments of the oncological process in a sample of adolescent survivors of childhood cancer $(\mathrm{N}=41)$. It will also explore whether this optimism is associated with coping in such situations as well as with health-related quality of life (HRQoL) in survival.

Results: Up to $70.7 \%$ of the sample referred to be optimistic in the worst situations of the illness. Those adolescents considered as "pessimistic" showed greater use of "selfblame" and "seek for spiritual support" strategies. However, it could not be observed a significant relationship between optimism and coping in the worst self-reported situations, and only one significant relationship was identified between optimism and the "physical well-being" dimension of HRQoL.

Conclusions: It could not be identified a clear effect of optimism displayed during cancer and HRQoL in survival period. Thus, it is possible that optimism might be a necessary condition to face difficulties in a more adaptative manner during the illness, but not sufficient to have a significant effect on resulting $H R Q o L$ after cancer remission. It is even probable that optimism may facilitate the occurrence of other variables that could have a more significant effect on HRQoL in

\section{Correspondencia:}

Carmina Castellano-Tejedor.

Hospital Universitario Vall d'Hebron de Barcelona. Área de Rehabilitación y Traumatología. Planta 1. Psicología.

Passeig Vall d'Hebron 119-129, 08035, Barcelona, España.

E-mail: ccastellano@vhebron.net, castellano.tejedor@yahoo.es 
tipo de variables que sí tengan un efecto más significativo sobre dicha CVRS en periodo de supervivencia. Son necesarios más trabajos en población infanto-juvenil para clarificar tales cuestiones.

Palabras clave: Adolescentes oncológicos en remisión, optimismo, afrontamiento, estudio descriptivo mediante encuesta. survivorship. More research with childhood population is needed to further clarify these relationships.

Keywords: Adolescent survivors of childhood cancer, optimism, coping, descriptive study.

\section{INTRODUCCIÓN}

El concepto de optimismo ha sido estudiado desde dos perspectivas teóricas diferentes, aunque no opuestas: la del optimismo disposicional de Scheier y Carver $^{(1)}$ y la del estilo explicativo de Peterson y Seligman ${ }^{(2)}$.

a. Optimismo como característica disposicional: Michael Scheier y Charles Car$\operatorname{ver}^{(1)}$, dos de los investigadores más conocidos en el campo del optimismo, lo definen como una característica disposicional que media entre los acontecimientos externos y la interpretación personal de los mismos. Desde esta perspectiva, el optimismo se relacionaría directamente con las expectativas de las personas hacia el futuro. Las personas optimistas tienen la expectativa o creencia estable y generalizada de que en la vida les ocurrirán mayoritariamente cosas positivas; mientras que los pesimistas creen que la vida les deparará cosas principalmente negativas. De este modo, se teoriza que cuando surgen dificultades, las expectativas favorables propias de los optimistas les llevan a incrementar los esfuerzos para alcanzar sus objetivos. Los pesimistas, en cambio, guiados por sus perspectivas desfavorables, reducen tales esfuerzos, por lo que hacen más difícil el que se vean cumplidos sus objetivos ${ }^{(3)}$.

Desde la aparición del concepto, se han desarrollado diversos estudios tratando de poner en relación el optimismo disposicional con diversos factores físicos y psicológicos. Así, por ejemplo, se ha demostrado que el optimismo es una variable que media en la manera que tienen las personas de enfrentarse a situaciones estresantes ${ }^{(3,4)}$. En este sentido, algunos trabajos indican que optimistas y pesimistas parecen utilizar estrategias de afrontamiento diferentes frente al estrés: las estrategias de los optimistas suelen estar más centradas en la solución del problema (p.e. planificación o reinterpretación positiva), mientras que las estrategias de los pesimistas suelen estar más orientadas hacia la evitación del problema o la desvinculación cognitiva y/o conductual del mismo (p.e. negación, distanciamiento mental, actividades distractoras). Estas diferencias, podrían estar detrás del hecho de que las personas optimistas, perciban mayor control sobre la situación estresante que los pesimistas y, de algún modo, esto reduzca su malestar emocional y la sintomatología física comúnmente asociada a altos niveles de estrés ${ }^{(5,6)}$. Por tanto, algunos investigadores han sugerido que el pesimismo podría asociarse a una peor calidad de vida en relación a la salud (en adelante, CVRS) ${ }^{(7)}$.

b. Optimismo como estilo explicativo: Christopher Peterson y Martin Seligman entienden que las expectativas que las personas tienen hacia acontecimientos futuros se relacionan íntimamente con cómo explican los acontecimientos del pasado ${ }^{(2,8)}$. El estilo explicativo hace referencia a las diferentes formas que tiene una persona de afrontar y explicarse a sí misma las situaciones que le han ocurrido en la vida. Según Peterson y Seligman ${ }^{(8)}$ los estilos expli- 
cativos se definen por tres características que pueden entenderse como dimensiones binarias:

1. Internalidad/externalidad: personalización o grado en que la persona se considera responsable de un suceso.

2. Estabilidad/inestabilidad: permanencia o grado en que la causa de un suceso se mantiene en el tiempo.

3. Globalidad/especificidad: persistencia o grado en que un aspecto de un suceso o de su explicación domina otros aspectos de la vida del individuo.

Basándonos en estas tres dimensiones y teniendo en cuenta los sucesos negativos, podemos decir que un mismo acontecimiento puede entenderse desde dos estilos explicativos diferentes. Así, el estilo explicativo optimista se caracteriza por la externalidad, la inestabilidad y la especificidad; mientras que el estilo explicativo pesimista se caracteriza por las dimensiones de internalidad, estabilidad y globalidad. En cuanto a los acontecimientos positivos, ocurre exactamente lo contrario. Los optimistas creen que los sucesos positivos tienen una causa interna, permanente y global, de manera que dependen de uno mismo, son duraderos y pueden afectar a todos los ámbitos de la vida; mientras que los pesimistas creen que tienen causas externas, temporales y específicas, es decir, asumen que los sucesos positivos no dependen de ellos, que son pasajeros y que sólo afectan a ámbitos muy concretos de su vida. Martin Seligman $^{(9)}$ explicaba que el tener un estilo explicativo u otro vendría determinado en gran medida por una conjunción de diferentes factores y circunstancias; desde factores genéticos y temperamentales, hasta las características propias del ambiente socio-familiar en que se desarrolla y evoluciona un individuo.
Dentro de este enfoque, existen diversos estudios que relacionan el optimismo como estilo explicativo y distintos valores de salud auto-percibida ${ }^{(10-12)} \mathrm{o}$ incluso diferencias en cuanto a longevidad ${ }^{(11,13)}$.

Considerando lo anterior, se hipotetiza que un individuo que supere satisfactoriamente en términos de adaptación una situación altamente aversiva, presentará uno de estos dos perfiles previamente comentados: o bien cierto rasgo de optimismo disposicional que le lleve a mantener la fe y/o confianza en que las cosas le van a ir mejor en el futuro; o bien cierto estilo explicativo optimista. Recordemos que esto último quiere decir: que no se considera responsable de lo sucedido (externalidad), que no piensa que va a estar en esa situación amenazante siempre (inestabilidad) y que no deja que ese suceso afecte todos y cada uno de los distintos aspectos de su vida (especificidad). Finalmente, indicar que desde la psicología positiva no se defiende un optimismo a ultranza, sino que éste sea flexible e inteligente, puesto que no se trata de la única forma válida para afrontar la vida y sus dificultades. Hay ocasiones en las que adoptar un pensamiento más negativo, pesimista, o simplemente más realista, puede resultar más $\operatorname{adaptativo}^{(9)}$.

Por lo que respecta al estudio del optimismo en el ámbito concreto de la psico-oncología, el marco teórico desde el que se fundamentan la mayor parte de trabajos, propone que la forma en que una persona acepta o se enfrenta a la enfermedad es susceptible de repercutir en cierta medida en su evolución. En este sentido, desde la psicología se ha tratado de contribuir a la investigación oncológica intentando encontrar relaciones entre variables psicológicas y la aparición, desarrollo y recuperación del cáncer ${ }^{(14)}$. El estudio del optimismo versus el pesimismo (la actitud de derrota en contraposición a la actitud de lucha), parece ser un 
factor importante en la evolución de las enfermedades en general y del cáncer en particular ${ }^{(15,16)}$. A pesar de que en los últimos años diversos trabajos han indicado una correlación positiva entre optimismo y supervivencia oncológica ${ }^{(17,18)}$, existen otros trabajos que no han confirmado dicha relación ${ }^{(19,20)}$.

Respecto al estilo explicativo optimista y el cáncer, el trabajo de Taylor y Brown del año 1988 resulta clave $^{(21)}$. En dicho documento se define lo que se conocería como "ilusiones positivas" frente a eventos desfavorables dentro de un estilo explicativo optimista. Se trataría de actitudes favorables y poco realistas que algunos individuos tienen respecto a sí mismos. Taylor y Brown establecieron tres categorías de ilusiones positivas: 1 . Una valoración al alza de las habilidades y/o capacidades de uno mismo, 2. El optimismo no-realista sobre el futuro y 3 . Las ilusiones de control. A día de hoy, sigue siendo controvertido el poder evaluar de forma fiable el grado en que las personas presentan este tipo de ilusiones positivas, así como el valorar el grado de influencia que dichas ilusiones pueden tener sobre el estado de salud mental de las personas que las experimentan. Desde el modelo de salud mental de Taylor y Brown ${ }^{(21)}$, se ha asumido que las creencias positivas en situaciones de adversidad, van a estar directamente relacionadas con el bienestar psicológico y que estas auto-evaluaciones positivas, aunque no sean estrictamente realistas, van a promover la salud mental. Para estos autores, el bienestar psicológico es entendido como la habilidad de sentirse bien con uno mismo, de ser creativo y/o productivo en las funciones que uno desempeña, así como la capacidad de mantener relaciones satisfactorias con otras personas y combatir eficazmente el estrés cuando esto sea necesario(21). Así, las ilusiones positivas podrían resultar particularmente útiles para ayudar a la gente a superar situaciones traumáticas o altamente adversas como accidentes graves, o también, enfermedades con riesgo potencial para la vida de la persona como sería el caso de muchas patologías oncológicas. En este sentido, Taylor y Brown ${ }^{(21,22)}$ describen que los pacientes oncológicos que han sido capaces de desarrollar o mantener este tipo de creencias o ilusiones positivas frente a los diversos estresores de su enfermedad, tienden a hacer uso de un afrontamiento más adaptativo y muestran menor malestar emocional y psicopatología que aquellos que no lo hacen.

Considerando todo lo anterior, el presente estudio parte de una conceptualización del optimismo como factor disposicional que tenderá a mantenerse en situaciones de adversidad, y se plantean los siguientes objetivos, a valorar en una muestra de adolescentes oncológicos en remisión:

(1) Evaluar los niveles de optimismo en los peores momentos de la enfermedad oncológica.

(2) Analizar la relación entre optimismo y las estrategias de afrontamiento llevadas a cabo en los peores momentos de la enfermedad.

(3) Analizar si existe relación entre dicho optimismo y la CVRS en periodo de remisión oncológica.

\section{MÉTODO}

Se trata de un estudio descriptivo mediante encuesta en el que se ha utilizado un diseño transversal, según la clasificación de Montero y León ${ }^{(23)}$.

\section{Participantes}

Se recogió información de un total de 41 adolescentes oncológicos en remisión de entre 13 y 20 años de edad $(M=17$, DS $=1,94)$ tratados en el Servicio de Onco- 
logía y Hematología Pediátrica del Hospital Universitario Vall d'Hebron de Barcelona que acudían a visitas de seguimiento en el mismo servicio. La mayoría de los adolescentes ( $\mathrm{n}=29 ; 70,7 \%)$ estaban cursando Educación Secundaria Obligatoria o BachiIler (desde $2^{\circ}$ de la ESO hasta $2^{\circ}$ de BachiIler). El número de familiares con los que vivía el adolescente (incluyéndose él mismo) oscilaba entre 2 y 6 personas $(M=3,85$, $\mathrm{DS}=0,88)$. El resto de características demográficas y clínicas se muestran en la tabla 1.

Criterios de inclusión y exclusión de la muestra

Los criterios de inclusión requerían que: (a) todos los participantes hubiesen sido diagnosticados de patología oncológica (excluyendo tumores del sistema nervioso central) a partir de los 8 años de edad en adelante, (b) que en el momento del estudio tuviesen entre 13 y 20 años (ambos incluidos), (c) que en el momento del estudio llevasen un periodo igual o superior a un 1 fuera de tratamiento oncológico, (d) que tuviesen una comprensión suficiente del castellano y/o catalán, (e) que tuviesen programada una visita de control en el centro hospitalario de referencia en el momento del estudio. Todos aquellos adolescentes con diagnóstico psicopatológico o alteración cognitiva previa a la enfermedad oncológica fueron excluidos del estudio.

Reclutamiento y recolección de datos

El periodo de reclutamiento y recolección de datos tuvo lugar entre mayo del 2009 y mayo del 2010. Tanto el procedimiento como las medidas del estudio fueron aprobadas por el comité de ética del Hospital Universitario Vall d'Hebron de Barcelona, España. Todos los procedimientos de la presente investigación cumplen con los principios de investigación ética
Tabla 1. Características demográficas y clínicas de la muestra $(N=41)$

\begin{tabular}{|c|c|c|c|}
\hline \multicolumn{2}{|l|}{ s. } & $\mathrm{n}$ & $\%$ \\
\hline \multicolumn{4}{|l|}{ Género } \\
\hline \multicolumn{2}{|l|}{ Hombre } & 26 & 63,4 \\
\hline \multicolumn{2}{|l|}{ Mujer } & 15 & 36,6 \\
\hline \multicolumn{4}{|c|}{ Nivel de estudios completado } \\
\hline \multicolumn{2}{|c|}{$2^{\circ}$ de ESO } & 2 & 4,9 \\
\hline \multicolumn{2}{|l|}{$3^{\circ}$ de ESO } & 5 & 12,2 \\
\hline \multicolumn{2}{|l|}{$4^{\circ}$ de ESO } & 8 & 19,5 \\
\hline \multicolumn{2}{|c|}{$1^{\circ}$ Bachiller LOGSE/COU } & 6 & 14,6 \\
\hline \multicolumn{2}{|c|}{$2^{\circ}$ Bachiller LOGSE/COU } & 8 & 19,5 \\
\hline \multicolumn{2}{|c|}{$1^{\circ}$ Grado ciclo formativo } & 5 & 12,2 \\
\hline \multicolumn{2}{|c|}{$2^{\circ}$ Grado ciclo formativo } & 4 & 9,8 \\
\hline \multicolumn{2}{|c|}{$1^{\circ}$ Licenciatura } & 2 & 4,9 \\
\hline \multicolumn{2}{|l|}{$2^{\circ}$ Licenciatura } & 1 & 2,4 \\
\hline \multicolumn{4}{|l|}{ Diagnóstico } \\
\hline \multicolumn{2}{|l|}{ Leucemia } & 14 & 34,1 \\
\hline \multicolumn{2}{|c|}{ Linfoma } & 18 & 43,9 \\
\hline \multicolumn{2}{|c|}{ Otros tumores sólidos } & 9 & 22 \\
\hline \multicolumn{4}{|c|}{ Tratamiento } \\
\hline \multicolumn{2}{|c|}{ Sólo quimioterapia } & 14 & 34,15 \\
\hline \multicolumn{2}{|c|}{ Sólo intervención quirúrgica } & 1 & 2,44 \\
\hline \multicolumn{2}{|c|}{ Terapia combinada ${ }^{a}$} & 26 & 63,41 \\
\hline \multicolumn{4}{|c|}{ Trasplante de médula ósea } \\
\hline \multicolumn{2}{|c|}{ Sí } & 11 & 26,8 \\
\hline \multicolumn{2}{|l|}{ No } & 30 & 73,2 \\
\hline \multicolumn{4}{|l|}{ Recaída } \\
\hline \multicolumn{2}{|l|}{ Sí } & 4 & 9,75 \\
\hline \multicolumn{2}{|l|}{ No } & 37 & 90,25 \\
\hline \multicolumn{4}{|c|}{ Aparición de segundas neoplasias } \\
\hline \multicolumn{2}{|l|}{ Sí } & 1 & 2,4 \\
\hline No & & 40 & 97,6 \\
\hline & Media & DS & Rango \\
\hline Edad (en años) & 17 & 1,94 & $13-20$ \\
\hline $\begin{array}{l}\text { Edad en el } \\
\text { diagnóstico (en } \\
\text { años) }\end{array}$ & 11,76 & 2,55 & $8-16$ \\
\hline $\begin{array}{l}\text { Años desde el } \\
\text { diagnóstico }\end{array}$ & 5,80 & 2,61 & $2-11$ \\
\hline
\end{tabular}

a Bajo este epígrafe se incluyen las combinaciones siguientes: quimioterapia + radioterapia, quimioterapia + intervención quirúrgica, quimioterapia + intervención quirúrgica + radioterapia, radioterapia + intervención quirúrgica. 
con humanos de la declaración de Helsinki y sus posteriores enmiendas. En todos los casos la participación fue voluntaria. A través de un consentimiento informado se aseguró la confidencialidad y el anonimato de los datos.

\section{Instrumentos}

Para medir el optimismo o predisposición generalizada hacia las expectativas de resultados positivos, se utilizó el Test de Orientación Vital revisado (Life Orientation Test-Revised; en adelante LOT-R), de Scheier, Carver y Bridges $^{(24)}$ según la adaptación española de Otero et al. ${ }^{(25)}$. El LOT-R puede administrarse de forma genérica o bien específica preguntando acerca de un evento concreto previamente seleccionado.

En la presente investigación se administró el LOT-R en formato específico. Para ello, se pedía a los participantes que tratasen de identificar lo que según su criterio fue el peor momento o situación vivida durante la enfermedad oncológica y que, centrándose es esos momentos, respondiesen en qué medida tendían a mantener expectativas positivas o negativas con respecto a los resultados del proceso y a su propio futuro. Al plantear un estudio transversal en lugar de longitudinal, la evaluación del optimismo durante el proceso oncológico tuvo que realizarse de forma retrospectiva.

La versión revisada del LOT consta de 6 ítems (más 4 de relleno) en los que se pregunta a los sujetos que indiquen el grado de acuerdo o desacuerdo con cada afirmación usando una escala Likert de 5 puntos (de $0=$ Muy en desacuerdo, a 4 = Muy de acuerdo). De los 6 ítems, 3 están redactados en sentido positivo y 3 en sentido negativo. Después de invertir las puntuaciones de los ítems redactados en sentido negativo, se suman todos los valores y se obtiene la puntuación total de optimismo que varía entre 0 y 24 puntos, considerándose que a mayor puntuación, mayor optimismo por parte del individuo. Los autores del instrumento proponen tomar como punto de corte una puntuación mayor o igual a 16 para considerar al individuo que responde el cuestionario como "optimista"(24,25). El LOT-R ha demostrado su utilidad en numerosos estudios tanto con población general como clínica, presenta unas adecuadas propiedades psicométricas y además, resulta de fácil y rápida administración (24-26). En nuestra muestra, no obstante, el Alfa de Cronbach fue de 0,61; algo inferior a la obtenida en el trabajo de Ferrando et al. (Alfa de Cronbach=0,78) (26). $^{(2)}$

Para la evaluación de las estrategias de afrontamiento se administró la Escala de Afrontamiento para Adolescentes (Adolescent Coping Scale; en adelante, ACS) de Frydenberg y Lewis ${ }^{(27,28)}$ en su adaptación española de Pereña y Seisdedos ${ }^{(29)}$. Este inventario de auto-informe consta de 80 ítems a partir de los cuáles se obtiene información acerca de la frecuencia de uso de 18 estrategias de afrontamiento que pueden agruparse en tres estilos básicos (ver la tabla 2): 1. Afrontamiento dirigido a la resolución del problema, 2. Afrontamiento en relación con los demás y 3. Afrontamiento improductivo ${ }^{(27,28)}$. De los 80 elementos de los que se compone el instrumento, 1 ítem es abierto y los 79 restantes son de respuesta cerrada en una escala Likert de 5 puntos (de $1=$ No me ocurre nunca o no lo hago, a $5=$ Me ocurre o lo hago con mucha frecuencia). Los valores Alfa fueron de 0,84 para el Afrontamiento dirigido a la resolución del problema, de 0,82 para el Afrontamiento improductivo y de 0,41 para el Afrontamiento en relación con los demás.

Los resultados por estrategia o por estilo básico de afrontamiento pueden interpretarse cualitativamente como se indica en la tabla 3. 


\section{Tabla 2. Relación de estrategias y estilos de afrontamiento del ACS}

\section{Estrategias de afrontamiento ( $\mathrm{n}^{0}$ de ítems)}

1. Buscar apoyo social (AS): (5 ítems)

2. Concentrarse en resolver el problema (REP): (5 ítems)

3. Esforzarse y tener éxito (ES): (5 ítems)

4. Preocuparse (PR): (5 ítems)

5. Invertir en amigos íntimos ( $\mathrm{Al})$ : (5 ítems)

6. Buscar pertenencia (PE): (5 ítems)

7. Hacerse ilusiones (HI): (5 ítems)

8. Estrategia de falta de afrontamiento (NA): (5 ítems)

9. Reducción de la tensión (RT): (5 ítems)

10. Acción social (SO): (4 ítems)

11. Ignorar el problema (IP): (4 ítems)

12. Autoinculparse (CU): (4 ítems)

13. Reservarlo para sí (RE): (4 ítems)

14. Buscar apoyo espiritual (AE): (4 ítems)

15. Fijarse en lo positivo ( $\mathrm{PO})$ : (4 ítems)

16. Buscar ayuda profesional (AP): (4 ítems)

17. Buscar diversiones relajantes (DR): (3 ítems)

18. Distracción física (FI): (3 ítems)

Estilos de afrontamiento (estrategias que lo componen)

1. Dirigido a la resolución del problema (DR, ES, FI, PO, REP)

2. Afrontamiento en relación con los demás $(A E, A I, A P, A S, P E, S O)$

3. Afrontamiento improductivo $(C U, H I, I P, N A, P R, R E, R T)$

Fuente: adaptado de Pereña y Seisdedos ${ }^{(29)}$.

Tabla 3. ACS: Interpretación de las puntuaciones (por escala $y / 0$ estilo de afrontamiento)

$$
\begin{array}{ll}
20-29 & \text { Estrategia no utilizada / estilo no utilizado } \\
30-49 & \text { Se utiliza raras veces } \\
50-69 & \text { Se utiliza algunas veces } \\
70-89 & \text { Se utiliza a menudo } \\
90-105 & \text { Se utiliza con mucha frecuencia }
\end{array}
$$

Para evaluar CVRS se utilizó el Cuestionario KIDSCREEN-52 ${ }^{(30)}$ en versión autoinformada. El KIDSCREEN consta de 52 ítems que se agrupan en 10 dimensiones de calidad de vida: 1. Bienestar físico,
2. Bienestar psicológico, 3. Estado de ánimo y emociones, 4. Auto-percepción, 5. Autonomía, 6. Relación con los padres y vida familiar, 7. Amigos y apoyo social, 8. Entorno escolar, 9. Aceptación social 
(ausencia de bullying) y 10. Recursos económicos. El rango de puntuación de todos los ítems es de 1 a 5. Sus puntuaciones pueden estandarizarse para cada dimensión $(M=50, D S=10)$ de forma que a mayores puntuaciones, mejor CVRS en la dimensión específica evaluada. El KIDSCREEN-52 cuenta con baremos europeos y españoles en función del género y la edad del adolescente. Este cuestionario ha demostrado ser un instrumento útil para valorar CVRS tanto en población pediátrica general, como en población pediátrica afecta de distintas enfermedades crónicas y/o con problemas de salud ${ }^{(31)}$. Además, el KIDSCREEN-52 resulta de fácil y rápida administración, no superando generalmente los 15-20 minutos. En nuestra muestra, todos los valores Alfa de Cronbach estuvieron por encima de 0,65 con la salvedad de la dimensión de Autonomía que obtuvo un Alfa de 0,47.

Los datos demográficos y médicos se recogieron a través de un protocolo ad hoc desarrollado para la presente investigación.

\section{Procedimiento}

Una vez aprobado el proyecto por el comité de investigación y ética del hospital de referencia se procedió al periodo de reclutamiento y recolección de datos.

A través del Registro Nacional de Tumores Infantiles (RNTI-SEOP) ${ }^{(32)}$ se identificó a los candidatos potenciales para participar en el estudio. Una vez identificados todos los supervivientes que cumplían criterios de inclusión (93 familias), se contactó telefónicamente con 47 familias (50,5\%) para explicarles el objetivo del estudio y pedirles colaboración. Las 46 familias restantes $(49,5 \%)$ o bien no fueron localizadas, o bien no tenían visita de seguimiento en el centro hospitalario de referencia durante la ejecución del estudio. Para las familias que aceptaron participar, se programó una sesión de evaluación el mismo día que tenían que acudir al hospital para las visitas de control médico del adolescente. En todos los casos, la participación en el estudio fue voluntaria. Finalmente, se contó con la participación de 41 familias. El índice de rechazo fue del $12,77 \%$ ( $n=6$ familias).

Los motivos de rechazo fueron en su mayor parte el no querer hablar del cáncer y recordar toda la experiencia vivida $(n=4$; $66,6 \%$ ). En un caso el argumento de rechazo fue la falta de tiempo $(16,7 \%)$ y en otro caso, la familia no acudió a la sesión de evaluación a pesar de haber aceptado telefónicamente en las dos ocasiones en las que fueron contactados $(16,7 \%)$.

La evaluación se llevó a cabo íntegramente por parte de la autora del presente trabajo, en una única sesión de alrededor de 45-60 minutos de duración, en un despacho del servicio de Oncología y Hematología Pediátrica del hospital. En primer lugar, la investigadora explicaba nuevamente el estudio y respondía a cualquier cuestión que pudiesen tener los participantes. Tras ello se solicitaba el consentimiento informado tanto por parte del adolescente como por parte de sus padres o representantes legales. Las variables demográficas y oncomédicas (incluyendo las secuelas derivadas del cáncer y su tratamiento) se recogían en presencia del adolescente y el/los progenitor/es en formato de entrevista semi-estructurada. Por último, se procedía a la administración de los distintos cuestionarios de evaluación sin la presencia de los progenitores.

\section{Análisis estadístico}

El análisis de los resultados se realizó con el programa estadístico SPSS versión 19.0 para Windows. Se Ilevaron a cabo:

a) Análisis descriptivos de la muestra para cada una de las variables con- 
templadas en el trabajo. Para ello, se han utilizado frecuencias, porcentajes, medias, desviaciones estándar, rangos (mínimo y máximo) y medianas.

b) Tests de Chi-cuadrado de Pearson para contrastar la hipótesis de independencia entre variables nominales.

c) Evaluación de las diferencias de medias de las variables continuas en función de las nominales grupales, a través de la prueba t de Student o la prueba homóloga no paramétrica $U$ de Mann-Whitney cuando la variable tenía dos categorías; o mediante análisis unidireccionales de la varianza cuando la variable tenía más de dos categorías.

d) Para estudiar el tipo y el grado de asociación existente entre variables continuas, se han llevado a cabo análisis de correlación lineal ( $r$ de Pearson) con nivel de significación bilateral. Para todos los resultados del presente trabajo, tan sólo se considerarán aquellas correlaciones que, además de ser estadísticamente significativas $(p<0,05)$, presenten un grado de correlación $\geq 0,3$.

La significación de todas las pruebas se ha considerado con un nivel de probabilidad del 5\% o inferior, indicando siempre la significación exacta que ofrecía el SPSS.

\section{RESULTADOS}

Objetivo 1: evaluar los niveles de optimismo en los peores momentos de la enfermedad oncológica (evaluación retrospectiva)

Según las puntuaciones del LOT-R, la gran mayoría de adolescentes $(n=29$, $70,7 \%$ ) afirmaron que se mantuvieron "optimistas" (>16 puntos) en los peores momentos de su enfermedad $(M=17,36$, $\mathrm{DS}=3,75$, rango $11-24$, mediana $=17$ ). No se observaron diferencias estadísticamente significativas en función del género, ni del tipo de diagnóstico o tratamiento recibido. La edad en el momento del diagnóstico tampoco mostró correlación con las puntuaciones de optimismo.

Objetivo 2. Analizar la relación entre optimismo y las estrategias de afrontamiento llevadas a cabo en los peores momentos de la enfermedad (evaluación retrospectiva)

En cuanto a los estilos de afrontamiento, el más usado por los adolescentes en los peores momentos de su enfermedad, fue el dirigido a la resolución del problema $(M=58,57, D S=13,47$, rango 31,80-87,80, mediana $=59)$. No obstante, sólo manifestaban hacer uso de éste algunas veces (véase la tabla 3 para la interpretación del valor obtenido). Tanto el estilo de afrontamiento improductivo $(M=47,30, D S=9,27$, rango $27,43-72,14$, mediana $=46,85)$ como el estilo de afrontamiento en relación con los demás $(M=45,82, D S=10,70$, rango 28,1773,50, mediana $=45,16$ ) fueron utilizados raras veces. La tabla 4 presenta los resultados descriptivos así como la preferencia de uso (de mayor a menor) de las distintas estrategias de afrontamiento evaluadas en referencia al peor momento o situación vivida durante el cáncer.

En los peores momentos, destaca claramente el uso de la estrategia de afrontamiento "fijarse en lo positivo" (se utiliza a menudo). A ésta le siguen las estrategias de afrontamiento de "preocuparse", "hacerse ilusiones" y "buscar diversiones relajantes" de las que los adolescentes manifestaban hacer uso algunas veces.

Se encontraron diferencias estadísticamente significativas en función del género, en el uso de la estrategia "ignorar el proble$\mathrm{ma}^{\prime \prime}$ en los peores momentos $\left(\mathrm{t}_{(39)}=2,596\right.$; $\mathrm{p}=0,013$, IC del 95\%: 2,70 a 21,78), ya que los chicos hacían mayor uso de esta estrategia $(M=40,58, D S=16,02)$, en com- 
Tabla 4. Estadísticos descriptivos y ordenación de las estrategias de afrontamiento utilizadas por los adolescentes en la peor situación vivida durante su enfermedad ( $N=41)$

\begin{tabular}{|c|c|c|c|c|}
\hline $\begin{array}{l}\text { Preferencia } \\
\text { de uso }\end{array}$ & $\begin{array}{c}\text { Estrategia } \\
\text { de afrontamiento }\end{array}$ & Media (DS) & $\begin{array}{c}\text { Rango } \\
(\min -\text { máx })\end{array}$ & Mediana \\
\hline $1^{\circ}$ & Fijarse en lo positivo ${ }^{a}$ & $72,07(16,80)$ & $40-100$ & 75,00 \\
\hline $2^{\circ}$ & Preocuparse $^{c}$ & $67,51(16,27)$ & $32-100$ & 68,00 \\
\hline $3^{\circ}$ & Hacerse ilusiones ${ }^{c}$ & $67,02(15,27)$ & $36-96$ & 64,00 \\
\hline $4^{\circ}$ & Buscar diversiones relajantes ${ }^{a}$ & $66,07(21,69)$ & $21-105$ & 63,00 \\
\hline $5^{\circ}$ & Concentrarse en resolver el problema ${ }^{a}$ & $59,90(17,37)$ & $20-88$ & 60,00 \\
\hline $6^{\circ}$ & Buscar pertenencia ${ }^{b}$ & $56,98(15,99)$ & $16-88$ & 52,00 \\
\hline $7^{0}$ & Esforzarse y tener éxito a & $55,22(17,41)$ & $20-92$ & 56,00 \\
\hline $\mathbf{8}^{\mathbf{o}}$ & Apoyo social ${ }^{b}$ & $54,44(14,08)$ & $36-100$ & 52,00 \\
\hline $9^{\circ}$ & Buscar ayuda profesional ${ }^{\text {b }}$ & $53,05(24,36)$ & $20-95$ & 55,00 \\
\hline $10^{\circ}$ & Reservarlo para sí c & $48,54(18,31)$ & $20-95$ & 45,00 \\
\hline $11^{\circ}$ & Invertir en amigos íntimos ${ }^{\text {b }}$ & $45,37(17,38)$ & $20-100$ & 44,00 \\
\hline $12^{\circ}$ & No afrontamiento $^{c}$ & $40,00(11,31)$ & $20-72$ & 40,00 \\
\hline $13^{\circ}$ & Distracción física a & $39,61(20,44)$ & $21-98$ & 35,00 \\
\hline $14^{\circ}$ & Reducción de la tensión c & $38,05(13,39)$ & $20-76$ & 36,00 \\
\hline $15^{\circ}$ & Ignorar el problema ${ }^{\mathrm{c}}$ & $36,10(15,55)$ & $20-80$ & 35,00 \\
\hline $16^{\circ}$ & Auto-inculparse ${ }^{c}$ & $33,90(15,06)$ & $20-75$ & 30,00 \\
\hline $17^{o}$ & Búsqueda de apoyo espiritual b & $33,78(14,39)$ & $20-90$ & 30,00 \\
\hline $18^{\circ}$ & Acción social ${ }^{b}$ & $31,34(10,49)$ & $20-70$ & 30,00 \\
\hline
\end{tabular}

a Estilo de afrontamiento de resolución del problema.

b Estilo de afrontamiento en relación con los demás.

c Estilo de afrontamiento improductivo.

paración con las chicas, que prácticamente no la usaban nunca $(M=28,33, D S=11,43)$.

La edad en el momento del diagnóstico mostró correlaciones estadísticamente significativas con el uso de la estrategia de "concentrarse en resolver el problema" $(r=0,420, p=0,006)$ y "reservarlo para sí" $(r=0,355, p=0,023)$.

Asimismo, se encontraron diferencias significativas en función del tipo de diagnóstico oncológico en la estrategia de "esforzarse y tener éxito" $\left(\mathrm{t}_{(30)}=-2,300\right.$; $p=0,029$, IC del 95\%: -24,75 a -1,47), Así, los adolescentes que habían sido diagnos- ticados de linfoma ( $n=18)$ hacían un mayor uso de esta estrategia en los peores momentos de su enfermedad $(M=61,11$, $\mathrm{DS}=14,89$ ), en comparación con los diagnosticados de leucemia ( $n=14 ; M=48,00$, $D S=17,32$ ). Sin embargo, no se encontraron diferencias en función del tipo de tratamiento recibido.

Por otra parte, se observaron diferencias en la frecuencia de uso de estrategias como "auto-inculparse" y "buscar apoyo espiritual" en función de si los adolescentes habían sido más o menos optimistas en los peores momentos. En este sentido, 
aquellos que consideraríamos "pesimistas" ( $n=12)$ según sus puntuaciones en el LOT-R, hicieron un mayor uso de la estrategia de "auto-inculpación" ( $M=43,33$, $\mathrm{DS}=14,51 ; \mathrm{t}_{(39)}=2,789 ; \mathrm{p}=0,008 ; \mathrm{IC}$ del $95 \%$ de 3,669 a 23,004), que aquellos que se mostraron más bien "optimistas" $(n=29)$ en los peores momentos de su enfermedad $(M=30,00, D S=13,69)$. Igualmente, los pesimistas demostraron hacer un uso significativamente superior de la estrategia "búsqueda de apoyo espiritual" $\quad\left(M=41,25, \quad D S=18,23 ; t_{(39)}=2,242\right.$; $\mathrm{p}=0,031$; IC del $95 \%$ de 1,034 a 20,087) en comparación con los optimistas $(M=30,69, D S=11,47)$. No obstante, en base a la interpretación cualitativa de las puntuaciones medias, en todos los casos se trataría de estrategias utilizadas raras veces.

Objetivo 3. Analizar si existe relación entre el optimismo en los peores momentos de la enfermedad y la CVRS en periodo de remisión oncológica

En la tabla 5 se presentan los descriptivos para las puntuaciones de CVRS.

\section{Tabla 5. Descriptivos de las puntuaciones de CVRS del KIDSCREEN-52 (N = 41 )}

\begin{tabular}{|c|c|c|c|c|c|}
\hline Dimensión & Género & Media (DS) & $\begin{array}{c}\text { Rango } \\
\text { (Min - Máx) }\end{array}$ & Mediana & p \\
\hline \multirow{2}{*}{ Bienestar físico } & Hombre $(n=26)$ & $50,34(5,38)$ & $42,53-64,30$ & 49,62 & \multirow{2}{*}{,003 } \\
\hline & Mujer $(n=15)$ & $43,47(8,30)$ & $30,57-64,30$ & 42,52 & \\
\hline \multirow{2}{*}{ Bienestar psicológico } & Hombre $(n=26)$ & $53,64(8,43)$ & $39,91-68,49$ & 53,13 & \multirow{2}{*}{$\begin{array}{c}\text { n.s. } \\
(, 059)\end{array}$} \\
\hline & Mujer ( $n=15)$ & $48,21(8,87)$ & $38,37-68,49$ & 47,11 & \\
\hline \multirow{2}{*}{ Estado de ánimo } & Hombre $(n=26)$ & $53,29(10,66)$ & $35,65-70,91$ & 54,02 & \multirow{2}{*}{ n.s. } \\
\hline & Mujer $(n=15)$ & $50,10(10,01)$ & $32,51-70,91$ & 49,09 & \\
\hline \multirow{2}{*}{ Auto-percepción } & Hombre $(n=26)$ & $47,81(4,09)$ & $37,85-60,11$ & 47,78 & \multirow{2}{*}{ n.s. } \\
\hline & Mujer $(n=15)$ & $47,72(7,80)$ & $41,83-69,78$ & 44,57 & \\
\hline \multirow{2}{*}{ Autonomía } & Hombre $(n=26)$ & $56,17(7,59)$ & $3,35-68,75$ & 56,27 & \multirow{2}{*}{,016 } \\
\hline & Mujer $(n=15)$ & $50,59(5,33)$ & $40,54-60,52$ & 50,77 & \\
\hline \multirow{2}{*}{$\begin{array}{l}\text { Relación con los padres y } \\
\text { vida familiar }\end{array}$} & Hombre $(n=26)$ & $51,27(8,98)$ & $35,66-65,87$ & 51,81 & \multirow{2}{*}{ n.s. } \\
\hline & Mujer $(n=15)$ & $50,91(10,21)$ & $36,98-65,87$ & 51,81 & \\
\hline \multirow{2}{*}{ Amigos y apoyo social } & Hombre $(n=26)$ & $58,27(9,15)$ & $42,20-71,46$ & 58,13 & \multirow{2}{*}{ n.s. } \\
\hline & Mujer $(n=15)$ & $55,37(8,49)$ & $36,81-71,46$ & 54,93 & \\
\hline \multirow{2}{*}{ Entorno escolar } & Hombre $(n=26)$ & $49,27(6,62)$ & $35,35-65,94$ & 48,61 & \multirow{2}{*}{ n.s. } \\
\hline & Mujer ( $\mathrm{n}=15)$ & $50,15(6,33)$ & $42,35-61,87$ & 48,61 & \\
\hline \multirow{2}{*}{$\begin{array}{l}\text { Aceptación social } \\
\text { (ausencia de bullying) }\end{array}$} & Hombre $(n=26)$ & $51,50(9,26)$ & $35,44-58,85$ & 58,84 & \multirow{2}{*}{ n.s. } \\
\hline & Mujer ( $n=15)$ & $49,79(10,26)$ & $31,08-58,85$ & 48,07 & \\
\hline \multirow{2}{*}{ Recursos económicos } & Hombre $(n=26)$ & $51,68(9,31)$ & $35,12-62,86$ & 50,84 & \multirow{2}{*}{ n.s. } \\
\hline & Mujer $(n=15)$ & $50,50(8,87)$ & $35,12-62,86$ & 49,27 & \\
\hline
\end{tabular}

n.s.: diferencias estadísticamente no significativas según la prueba $t(p>, 05)$. 
Como puede observarse, las puntuaciones medias para todas las dimensiones del KIDSCREEN-52 se encuentran en torno al valor promedio poblacional $(50 \pm 10)$. Sin embargo, los chicos obtuvieron puntuaciones medias significativamente más altas $(p=0,003)$ en la dimensión "bienestar físico" (50,34 vs. 43,47). Igualmente, estas diferencias se reflejaban en base a su clasificación según los baremos poblacionales del instrumento, en donde la puntuación media de los chicos se encontraría en el percentil 50 y la de las chicas en torno al percentil 30. Aunque en la dimensión "bienestar psicológico" las diferencias en función del género no llegaron a alcanzar el criterio de significación estadística $(p=0,059)$, atendiendo a los baremos poblacionales podemos observar que la media de los chicos se encontraría alrededor del percentil 75 y la de las chicas alrededor del percentil 50.

Por último, las puntuaciones medias obtenidas en la dimensión "autonomía" situaban a los chicos en el percentil 75 del baremo poblacional $(M=56,17)$, y aproximadamente en el 60 a las chicas $(M=50,59)$, siendo en este caso la diferencia estadísticamente significativa $(p=0,016)$.

No se encontraron diferencias estadísticamente significativas para ninguna de las 10 dimensiones del KIDSCREEN en función del tipo de diagnóstico oncológico. La edad actual, la edad en el momento del diagnóstico oncológico, o el tiempo transcurrido desde el mismo tampoco mostró relación con las puntuaciones de ninguna dimensión de CVRS evaluada. Sin embargo, sí existían diferencias estadísticamente significativas en función del tipo de tratamiento recibido (terapia combinada vs. sólo quimioterapia) en la dimensión de "auto-percepción" ( $U=85,000$, $\mathrm{p}=0,013)$; siendo los que recibían únicamente quimioterapia $(n=12)$, los que presentaban puntuaciones promedio más altas $(M=52,36, D S=8,00)$ en comparación con los que recibían terapia combinada $(n=28 ; M=45,75, D S=3,74)$. Si nos fijamos en el baremo poblacional del KIDSCREEN, las puntuaciones medias de aquellos adolescentes que recibieron únicamente quimioterapia se encontrarían en el percentil 75 , y las de aquellos que recibieron terapia combinada estarían en un percentil $<50$.

El optimismo en los peores momentos de la enfermedad oncológica tan solo correlacionó significativamente con el "bienestar físico" ( $r=0,320, p=0,041)$. No se encontraron diferencias estadísticamente significativas en ninguna dimensión de CVRS en función de si se era más bien optimista (LOT-R $\geq 16$ puntos) o por el contrario, pesimista (LOT-R $<16$ puntos) en el peor momento o situación vivida durante el cáncer.

\section{DISCUSIÓN Y CONCLUSIONES}

La presente investigación tenía tres objetivos: (1) evaluar los niveles de optimismo en los peores momentos de la enfermedad oncológica, (2) analizar la relación entre el optimismo y las estrategias de afrontamiento llevadas a cabo en los peores momentos de la enfermedad y (3) analizar la relación entre el optimismo y la CVRS resultante en periodo de remisión oncológica.

Respecto al primer objetivo, cabe destacar que la mayoría de adolescentes $(70,7 \%)$ manifestaron permanecer optimistas en los peores momentos de su enfermedad, con independencia del curso de la misma. Así, no sólo el género no supuso ninguna diferencia significativa sobre los niveles de optimismo indicados, sino que tampoco lo hizo ninguna de las variables oncomédicas contempladas en el presente trabajo. Uno de los antecedentes más directamente relacionados con nuestro trabajo sería el estudio de Grootenhuis y Last $^{(33)}$. Estos autores estudiaron dos grupos de niños con cáncer (de entre 8 y 18 
años) en distintas fases de la enfermedad oncológica. Un grupo estaba formado por pacientes oncológicos con pocas perspectivas de supervivencia y el otro, por niños y adolescentes en fase de remisión. En su trabajo, Grootenhuis y Last no encontraron diferencias significativas entre grupos en lo que respecta a niveles de ansiedad y depresión y, lo que es más importante para el objetivo que aquí nos concierne, observaron que ambos grupos mantenían expectativas positivas sobre el curso de la enfermedad (caracterizadas por un nivel de esperanza, confianza y optimismo comparables). Así pues, también en nuestra muestra hemos podido observar una mayoría de adolescentes que decían mantenerse optimistas en los peores momentos de su enfermedad y confiaban en que el futuro les depararía cosas mejores. Aunque es posible que al preguntar a los adolescentes sobre el peor momento o situación que vivieron durante su enfermedad, el tiempo transcurrido desde tal evento influya en su respuesta, creemos que en un estudio retrospectivo transversal como el que nos ocupa, el hecho de preguntar por un momento de alto impacto emocional (peor momento o situación vivida durante la enfermedad) ayuda a anclar el recuerdo y está menos sujeto a distorsiones de la memoria, que el preguntar por momentos de intensidad emocional moderada-baja, o promediar su malestar durante todo el proceso oncológico.

Cabe señalar, no obstante, que en estos resultados también podrían influir distintos factores como, por ejemplo, el nivel de maduración cognitiva. Aunque la integración de las diversas representaciones del self comienza a emerger hacia los 9-10 años y culmina al final de la adolescen$\mathrm{cia}^{(34)}$, este proceso no es igual para cada adolescente y el nivel de conocimiento que tengan de su enfermedad, así como de su gravedad o posibles implicaciones puede ser muy variopinto. Quizás incluso pueda tener lugar un cierto "optimismo no realista" caracterizado por un sesgo cognoscitivo que hace que el adolescente distorsione ligeramente la realidad y perciba menos peligro del que realmente hay (en línea con el optimismo explicativo y los trabajos de Taylor y Brown referidos en la introducción del presente trabajo) ${ }^{(21,22)}$. Sin embargo, esto no es necesariamente negativo, puesto que en cierto modo puede ejercer un papel protector, ayudando a disminuir el malestar, que podría llegar a ser excesivo en tales situaciones y favoreciendo así, un tipo de afrontamiento más adaptativo.

En línea con lo anterior, en nuestra muestra de adolescentes ha podido observarse una preferencia por el estilo de afrontamiento de resolución del problema y en concreto, por la estrategia de "fijarse en lo positivo", una de las claramente más utilizadas. Sin embargo, cabe indicar que en términos cualitativos manifestaban hacer uso de este estilo de afrontamiento activo algunas veces, por lo que aunque fuese el preferido, su uso no resultó ser demasiado elevado. Asimismo, el estilo improductivo y el afrontamiento en relación con los demás eran usados prácticamente por igual y con muy poca diferencia con respecto al estilo de afrontamiento de resolución del problema (manifestaron usarlos raras veces). Así, podemos ver que la segunda estrategia más usada por los adolescentes fue "preocuparse" y en tercer lugar, "hacerse ilusiones"; ambas pertenecientes a un estilo de afrontamiento improductivo. Aunque en nuestro estudio se preguntara por el afrontamiento en el peor momento de la enfermedad, este mismo patrón de preferencia de estilos de afrontamiento ha sido observado en población general sana de su misma edad(27-29), e incluso en adolescentes oncológicos en remisión ${ }^{(35)}$; por lo que cabría valorar si la situación concreta de la enfermedad oncológica va a suponer la puesta en marcha de una se- 
rie de estrategias de afrontamiento extraordinarias por parte de los adolescentes, o si simplemente, van a hacer aquello que suelen hacer de modo general frente a los distintos problemas que les van surgiendo en su día a día.

En la mayoría de investigaciones de psico-oncología, las estrategias que se han mostrado como más adaptativas frente a la enfermedad oncológica han sido las de tipo activo y las interpersonales; bien sea porque predisponen a asumir una actitud activa y participativa en el tratamiento, o en la toma de decisiones médicas; o bien por movilizar recursos de tipo social buscando apoyo emocional e informativo por parte del entorno sociofamiliar $^{(36)}$. Por otra parte, las estrategias de desvinculación cognitivo-conductual (improductivo) aparecen como menos eficaces en general, puesto que en algunos casos pueden dificultar la aceptación de la realidad y la elaboración del problema a nivel cognitivo y emocional por parte del paciente ${ }^{(37)}$. Pese a todo, hay que tener presente una serie de aspectos; en primer lugar, la mayoría de trabajos en esta línea provienen del estudio de pacientes oncológicos adultos y extrapolar los resultados a población infanto-juvenil supone serias limitaciones por las características idiosincrásicas que este periodo evolutivo implica $^{(38)}$. Además, Lazarus ${ }^{(39)}$ ya indicaba que no es correcto hablar de estrategias buenas o malas en términos absolutos, sino que la adecuación de éstas siempre deberá valorarse en función de las características de la situación estresante y de las características personales del individuo que le haga frente. En el ámbito de la psico-oncología se ha hablado de la utilidad de las estrategias de desvinculación cognitivo-conductual en diversos trabajos $^{(40,41)}$. En ellos, se postulaba la utilidad de estrategias tales como la negación o la represión emocional, como elementos que, puntualmente, pueden favorecer el seguir centrado en una serie de objetivos relacionados con el tratamiento o las rutinas cotidianas y no dejar lugar a la tristeza y/o la depresión, que en muchos casos podría llegar a ser incapacitante o interferir con las pautas médicas ${ }^{(42)}$. En lo referente específicamente a psicooncología pediátrica, Phipps et al. ${ }^{(43)}$ han señalado que el afrontamiento defensivo, de negación o represivo, podría jugar un importante papel en el mantenimiento de la funcionalidad de los niños y adolescentes con cáncer, favoreciendo su capacidad de ajuste durante todo el proceso oncológico. Estos autores, incluso Ilegan a afirmar que el hacer uso de un estilo de afrontamiento de desvinculación o represión emocional, no supone ningún coste en términos de salud física o psicológica en periodo de supervivencia. Aunque no concluyen que este estilo de afrontamiento sea el único camino, sí aseguran que es un camino más hacia la resiliencia en este tipo de población.

Por lo que respecta a las diferencias entre chicos y chicas, no hemos podido observar una preferencia de unas estrategias de afrontamiento frente a otras en función del género, tal y como se ha señalado en la población general ${ }^{(29)}$. La única diferencia encontrada fue en el caso de la estrategia "ignorar el problema", que al igual que en los valores de referencia poblacional del ACS, resultó más usada por parte de los chicos. Una posible explicación a esta diferencia, contrastada en algunos trabajos realizados en población con enfermedad hematológica, es que las chicas sean más propensas a expresar su malestar emocional o a comunicarlo a otros, haciendo un mayor uso de estrategias como la "ventilación emocional", o la "reinterpretación positiva"; por lo tanto, la negación sería menos elegida que en el caso de los chicos $^{(44)}$. Recordamos que en el presente trabajo se preguntaba acerca del peor momento, por lo que la comparación con 
la población general tiene validez en la medida en que entendamos las estrategias de afrontamiento como un patrón de respuesta cognitivo-conductual más o menos estable, que tenderá a repetirse en las distintas situaciones estresantes que puedan presentarse. Puede especularse que al preguntar por el peor momento, como a éste se le supone una alta demanda a nivel de recursos de afrontamiento, los adolescentes pusiesen en marcha más estrategias de afrontamiento y más variadas, o que incluso redistribuyesen la frecuencia de uso de las habituales, por lo que las diferencias típicas de cada género que se reflejarían en una situación menos demandante, podrían haber quedado diluidas dando lugar a este patrón de afrontamiento más homogéneo.

Algunos trabajos han señalado que los optimistas y los pesimistas parecen utilizar estrategias de afrontamiento diferentes frente al estrés. En este sentido, ha sido descrito que por regla general, los optimistas prefieren las estrategias de tipo activo centradas en la solución del problema, mientras que los pesimistas harían un mayor uso de estrategias de desvinculación cognitivo-conductual ${ }^{(3,4)}$. En nuestro trabajo, el ser más bien optimista o más bien pesimista, también apareció relacionado con la preferencia de uso de unas estrategias sobre otras. Así, los pesimistas manifestaban hacer un uso significativamente superior de la estrategia de afrontamiento de "auto-inculparse" y de la de "búsqueda de apoyo espiritual", en comparación con los optimistas.

Por último, la edad también parece determinar en cierta medida el tipo de estrategias elegidas en nuestra muestra; puesto que a mayor edad, los adolescentes mostraban mayor preferencia por el uso de estrategias centradas en la solución del problema ("concentrarse en resolver el problema") y algunas de desvinculación como el "reservarlo para sí". Aunque los resultados siguen siendo contradictorios ${ }^{(45)}$, la mayoría de trabajos indican que a mayor edad, mayor repertorio conductual y mayor tendencia a combinar diferentes estrategias de afrontamiento(27). Además, también pudo observarse que los adolescentes diagnosticados de linfoma hacían un uso significativamente superior de la estrategia de "esforzarse y tener éxito", en comparación con los adolescentes que padecían leucemia u otros tumores sólidos. Probablemente, esto se deba a las características del tratamiento que implica un linfoma, sobre todo de tipo Hodgkin, que en general, suele ser más demandante en lo que a duración y complejidad del tratamiento se refiere ${ }^{(46)}$.

Para el caso concreto de la CVRS, ha podido observarse que las puntuaciones se hallan dentro del promedio poblacional. Con la excepción de los tumores en el sistema nervioso central (excluidos de nuestro estudio); que son uno de los grupos diagnósticos con mayor número de secuelas y de mayor severidad, la literatura indica que la CVRS en periodo de supervivencia suele ser peor entre aquellos adolescentes diagnosticados de tumores óseos, leucemias mieloides y linfomas Hodgkin ${ }^{(47-50)}$. Sin embargo, en nuestro trabajo estas diferencias en función del tipo de cáncer no han podido observarse, quizás, por el escaso tamaño de la muestra y la baja representatividad de algunos diagnósticos oncológicos. La única diferencia significativa ha sido observada en la dimensión "autopercepción" para el grupo de adolescentes que recibieron terapia combinada. Quizás, la mayor movilización de recursos terapéuticos para combatir el cáncer ha podido suponer un cierto efecto de detrimento sobre la percepción de uno mismo y/o su propio estado de salud. No obstante, no hay que olvidar que las puntuaciones medias siguen estando dentro del promedio poblacional normal.

En los últimos años, la investigación acerca de los recursos psicológicos que 
favorecen la salud y el bienestar de las personas ha experimentado un aumento considerable ${ }^{(50,51)}$. En este sentido, el estudio del optimismo ha sido uno de los aspectos más ampliamente estudiados ${ }^{(16,52)}$. En uno de los trabajos clásicos de Scheier y Carver ${ }^{(1)}$ con un grupo de estudiantes de la población general, se indicaba que las personas consideradas "optimistas" al inicio de su estudio, eran también las que señalaban tener menos síntomas de malestar físico tanto en la primera evaluación llevada a cabo, como en evaluaciones posteriores. En otro trabajo de los mismos autores, pero en este caso con pacientes sometidos a una operación de by-pass, se encontró que el optimismo ejercía un fuerte y destacado efecto sobre el bienestar físico y psicológico de los pacientes; tanto antes, como inmediatamente después de la operación. Asimismo, los optimistas mostraron tener una recuperación más rápida y presentar un menor número de complicaciones médicas, que aquéllos que podrían considerarse más bien "pesimistas"(53).

En el ámbito específico de la psicooncología, estos mismos autores obtuvieron resultados similares con mujeres con cáncer de mama. Así, observaron que el optimismo manifestado por éstas, correlacionaba de forma negativa con sus niveles de malestar emocional y su angustia en el momento del diagnóstico ${ }^{(54)}$. No tenemos conocimiento de trabajos que hayan valorado el optimismo de pacientes oncológicos pediátricos y lo hayan puesto en relación con la CVRS de éstos en periodo de supervivencia. Sin embargo, en el trabajo de Michel y colaboradores con población pediátrica superviviente a un cáncer ${ }^{(55)}$, se indicaba que aunque el optimismo estaba relacionado significativamente con el benefit finding (beneficios percibidos) de esta población, éste no mostraba ninguna relación con su CVRS en periodo de remisión oncológica. Cabe tener presente que en dicho trabajo se tomó la medida disposicional genérica de optimismo y no la situacional como en nuestro caso.

En nuestro trabajo hemos podido observar una correlación positiva entre el optimismo manifestado por los adolescentes en los peores momentos de su enfermedad y la dimensión de "bienestar físico" de su CVRS en periodo de remisión. Estos resultados podrían ir en la línea de lo descrito por Scheier y Carver ${ }^{(1,53,54)}$, donde se indicaba que el optimismo juega un claro efecto favorecedor y potenciador del bienestar de los individuos. Sin embargo, su relación con la CVRS en periodo de supervivencia, no ha sido tan importante como cabría esperar, pues no ha mostrado correlación significativa con dimensiones tales como el "bienestar psicológico" o el "estado de ánimo". Es posible por tanto, que el optimismo situacional no sea un buen predictor de la CVRS posterior de esta población. Si bien es cierto que éste puede jugar un importante papel protector durante la enfermedad, su efecto no tiene por qué tener un reflejo en el periodo de remisión oncológica. Podría ser que el optimismo situacional fuese una condición necesaria para sobrellevar la enfermedad de un modo más adaptativo, pero quizás no es suficiente como para suponer una mayor CVRS una vez el cáncer ha remitido. Incluso es probable que el optimismo situacional pueda facilitar la ocurrencia de otro tipo de variables que sí tengan un efecto más significativo sobre dicha CVRS en periodo de supervivencia. A pesar de que en la literatura científica el optimismo se ha considerado tanto en su versión genérica disposicional como situacional, apenas existen trabajos que, como el nuestro, analicen la influencia del optimismo situacional en ámbito clínico ${ }^{(16)}$.

\section{Limitaciones}

La principal limitación de nuestro trabajo es el haber tenido que emplear 
medidas retrospectivas para valorar optimismo y estrategias de afrontamiento ligadas a los peores momentos experimentados durante el cáncer. Si bien es cierto que un diseño longitudinal hubiese sido más recomendable para nuestros objetivos, pensamos que al preguntar acerca del peor momento y/o situación vivida durante el cáncer, el sesgo de memoria es reducido al mínimo puesto que a tal hito temporal se le supone un elevado impacto emocional y esto suele tener un efecto muy marcado sobre el recuerdo, lo que reduciría considerablemente la posible distorsión de la memoria(56). Así, uno de los hechos que más Ilama la atención respecto a las memorias autobiográficas de hechos traumáticos es que los individuos suelen manifestar que son capaces de recordar esos sucesos como si acabasen de ocurrir, aparentando ser inmunes al deterioro producido por el paso del tiempo ${ }^{(56)}$. Las denominadas memorias vívidas (o flashbulb memories) consisten en memorias sobre hechos traumáticos que han tenido una importante repercusión personal y social ${ }^{(56,57)}$. Así, podemos pensar que en nuestra muestra de supervivientes oncológicos, un efecto similar a este tipo de recuerdo puede estar presente al preguntar sobre el peor momento $y / 0$ situación vivida durante su enfermedad. Sin embargo, también se ha descrito que al analizar tales recuerdos al detalle, ciertos aspectos que los individuos pueden dar por exactos, hayan sido "creados" posteriormente ${ }^{(57,58)}$. En este sentido, Schmidt ${ }^{(59)}$ encontró que los hechos centrales de un suceso traumático se recuerdan con más consistencia que los periféricos, pero que los recuerdos sobre este suceso contenían abundantes errores procedentes de una inapropiada reconstrucción de los hechos debida al paso del tiempo. No obstante, de cara a valorar cómo la experiencia potencialmente traumática puede estar afectando a la CVRS experimentada en la actualidad, no nos interesa tanto lo que sucedió realmente de forma fidedigna, sino el recuerdo que las personas tienen sobre lo que sucedió, que será lo que en última instancia determine las explicaciones y vivencias del suceso que refiera la persona al evocarlo.

Agradecimientos: Esta investigación se pudo realizar gracias a la beca de Formación de Personal Investigador FPI 00286 de la Universitat Autònoma de Barcelona y a la financiación recibida por parte del Ministerio de Ciencia e Innovación PSI201129807-C03-01/PSIC. Asimismo, nada de esto hubiese sido posible sin la colaboración voluntaria y desinteresada de todos los adolescentes entrevistados y de sus padres.

\section{REFERENCIAS BIBLIOGRÁFICAS}

1. Scheier MF, Carver CS. Optimism, coping and health: Assessment and implications of generalized outcome expectancies. Health Psychol 1985;4:219-47.

2. Peterson C, Seligman ME. Casual explanations as a risk factor for depression: Theory and evidence. Psychol Rev 1984;91:347-74.

3. Carver CS, Connor-Smith J. Personality and coping. Annu Rev Psychol, 2010; 61: 679-704. Doi: 10.1146/annurev. psych.093008.100352.

4. Chico-Librán E. Optimismo disposicional como predictor de estrategias de afrontamiento. Psicothema 2002;4:544-50.

5. Chang EC, Maydeu-Olivares A, D'Zurilla TJ. Optimism and pessimism as partially independent constructs: Relationship to positive and negative affectivity and psychological well-being. Pers Indiv Dif 1997;23:433-40.

6. Chang EC. Dispositional optimism and primary and secondary appraisal of a stressor: controlling for confounding influences and relations to coping and psychological and physical adjustment. J Pers Soc Psychol 1998;74:1109-20. 
7. Martínez-Correa A, Reyes del Paso G, García-León AJ, González-Jareño MI. Optimismo/Pesimismo disposicional y estrategias de afrontamiento del estrés. Psicothema, 2006;18:66-72.

8. Peterson C, Seligman ME. Explanatory style and illness. J Pers 1987;55:237-65.

9. Seligman ME. Aprenda optimismo. Madrid: Grijalbo, 1998.

10. Peterson C, Seligman ME, Vaillant GE. Pessimistic explanatory style is a risk factor for physical illness: A thirty-five-year longitudinal study. J Pers Soc Psychol 1988;55:23-7.

11. Reed GM, Kemeny ME, Taylor SE, Wang HY, Visscher BR. Realistic acceptance as a predictor of decreased survival time in gay men with AIDS. Health Psychol 1994;13:299307. Doi:10.1037/0278-6133.13.4.299

12. Reed GM, Kemeny ME, Taylor SE, Wang HY, Visscher BR. Negative HIV-specific expectancies and AIDS-related bereavement as predictors of symptom onset in asymptomatic HIV-positive gay men. Health Psychol 1999;18:354-63. Doi:10.1037/02786133.18.4.354

13. Maruta T, Colligan RC, Malinchoc M, Offord KP. Optimism-pessimism assessed in the 1960s and self-reported health status 30 years later. Mayo Clinic Proc 2002;77:748-53. Doi:10.4065/77.8.748

14. Dahl AA. Link between personality and cancer. Future Oncol 2010;6:691-707. Doi10.2217/fon.10.31

15. Aspinwall LG, Tedeschi RG. The value of positive psychology for health psychology: Progress and pitfalls in examining the relation of positive phenomena to health. Ann Behav Med 2010;39: 4-15. Doi: 10.1007/ s12160-009-9153-0.

16. Scheier MF, Carver CS, Bridges MW. Optimism, pessimism, and psychological wellbeing. En: Chang EC, editor. Optimism and pessimism: Implications for theory, research, and practice. Washington DC: American Psychological Association, 2001.p.189-216
17. Allison PJ, Guichard C, Fung K, Gilain L. Dispositional optimism predicts survival status 1 year after diagnosis in head and neck cancer patients. J Clin Oncol 2003;21:543-8. Doi: 10.1200/ JCO.2003.10.092

18. Rasmussen HN, Scheier MF, Greenhouse JB. Optimism and physical health: A meta-analytic review. Ann Behav Med 2009;37:239-56. Doi:10.1007/s12160009-9111-x.

19. Coyne JC, Pajak TF, Harris J, Konski A, Movsas B, Ang K, et al. Emotional wellbeing does not predict survival in head and neck cancer patients: A Radiation Therapy Oncology Group study. Cancer 2007;110:2568-75. Doi:10.1002/ cncr.23080

20. Schofield P, Ball D, Smith JG, Borland R, O'Brien P, Davis S, Olver I, Ryan G, Joseph D. Optimism and survival in lung carcinoma patients. Cancer 2004;100:1276-82.

21. Taylor SE, Brown J. Illusion and well-being: A social psychological perspective on mental health. Psychol Bull 1988;103:193210. Doi:10.1002/cncr.20076

22. Taylor SE, Kemeny ME, Reed GM, Bower JE, Gruenewald TL. Psychological resources, positive illusions, and health. Am Psychol 2000;55:99-109. Doi: 10. 1037//0003-066X.55.1.99

23. Montero I, León OG. A guide for naming research studies in Psychology. Int J Clin Health Psychol 2007;7:847-62.

24. Scheier MF, Carver CS, Bridges MW. Distinguishing optimism from neuroticism (and trait anxiety, self-mastery, and self-esteem): A reevaluation of the Life Orientation Test. J Pers Soc Psychol 1994;67:1063-78.

25. Otero JM, Luengo A, Romero E, Gómez JA, Castro C. Psicología de personalidad. Manual de prácticas. Barcelona: Ariel Practicum, 1998.

26. Ferrando PJ, Chico E, Tous J. Propiedades psicométricas del test de optimismo Life Orientation Test. Psicothema, 2002;14:673-80. 
27. Frydenberg E, Lewis R. Boys play sport and girls turn to others: age, gender and ethnicity as determinants of coping. J Adolesc 1993a;16:253-66.

28. Frydenberg E, Lewis R. Adolescent Coping Scale: handbook. Melbourne: Australian Council for Educational Research, 1993b.

29. Pereña J, Seisdedos N. Manual ACS: escalas de afrontamiento para adolescentes. Madrid: Ediciones TEA, 1996.

30. Ravens-Sieberer U, Gosch A, Rajmil L, Erhart M, Bruil J, Duer W, et al. The European KIDSCREEN Group. KIDSCREEN-52 quality-of-life measure for children and adolescents. Expert Rev Pharmacoecon Outcomes Res 2005;5:353-64. Doi: 10.1586/14737167.5.3.353.

31. Ravens-Sieberer U, Gosch A, Rajmil L, Erhart M, Bruil J, Power M, et al. The European KIDSCREEN Group. The KIDSCREEN-52 quality of life measure for children and adolescents: Psychometric results from a cross-cultural survey in 13 European countries. Value Health 2008;11:654-58. Doi: 10.1111/j.15244733.2007.00291.x.

32. Peris-Bonet R, García A, Muñoz F, Pardo E, Comisión científica de colaboradores de la SEOP. Registro Nacional de Tumores Infantiles (Informe estadístico RNTI-SEOP). Estadísticas básicas 1980-2006. Valencia: Universidad de Valencia, 2007.

33. Grootenhuis MA, Last BF. Children with cancer with different survival perspectives: Defensiveness, control strategies and psychological adjustment. Psychooncology 2001;10:305-14.

34. Harter S. The construction of the self. A developmental perspective. London: Guildford Press, 1999.

35. Castellano C. Papel de las estrategias de afrontamiento y la actividad física en la calidad de vida de adolescentes oncológicos en remisión. Trabajo de investigación no publicado, tesina, Universidad Autónoma de Barcelona, Bellaterra, 2008.
36. Koehler $M$, Koenigsmann $M$, Frommer J. Coping with illness and subjective theories of illness in adult patients with haematological malignancies: Systematic review. Crit Rev Oncol Hematol, 2009;69:237-57. Doi:10.1016/j.critrevonc.2008.09.014.

37. Kupst MJ. Coping with pediatric cancer: theoretical and research perspectives. En: Bearison DJ Mulhern RK, editors. Pediatric Psycho-oncology: Psychological perspectives on children with cancer. New York: Oxford University Press, 1994.p.45-60

38. Barr RD. The adolescent with cancer. Eur J Cancer 2001;37:1523-7.

39. Lazarus RS. Coping Strategies. En: McHugh S, Vallis TM, editors. Illness behavior. A multidisciplinary model. New York: Plenum Press,1986.p.303-8

40. Morley C. The use of denial by patients with cancer. Prof Nurse 1997;12:380-381.

41. Stephenson PS. Understanding denial. Oncol Nurs Forum 2004;31:985-8.

42. Vos MS, de Haes JC. Denial in cancer patients, an explorative review. Psychooncology 2007;16:12-25. Doi: 10.1002/pon.1051

43. Phipps S, Steele RG, Hall K, Leig L. Repressive adaptation in children with cancer: A replication and extension. Health Psychol 2001;20:445-51. Doi:10.1037/0278-

44. Jonassaint CR, Jonassaint JC, Stanton MV, de Castro LM, Royal CD. Clinical and sociodemographic factors predict coping styles among adults with sickle cell disease. J Natl Med Assoc 2010;102:1045-9.

45. Barrón RG, Castilla IM, Casullo MM, Verdú JB. Relación entre estilos y estrategias de afrontamiento y bienestar psicológico en adolescentes. Psicothema 2002;14:363-8.

46. Ellis JA. Psychosocial adjustment to cancer treatment and other chronic illnesses. Acta Paediatr 2000;89:134-6. Doi: 10.1111/j.1651-2227.2000.tb01202.x

47. Zebrack BJ, Chesler MA. Quality of life in childhood cancer survivors. Psychooncology 2002;11:132-41. Doi: 10.1002/pon.569 
48. Zeltzer LK, Recklitis C, Buchbinder D, Zebrack B, Casillas J, Tsao JC, et al. Psychological status in childhood cancer survivors: A report from the Childhood Cancer Survivor Study. J Clin Oncol 2009;27:2396404. Doi: 10.1200/JCO.2008.21.1433.

49. Bisegger C, Cloetta B, von Rueden U, Abel T, Ravens-Sieberer $U$, The European KIDSCREEN Group. Health-related quality of life: gender differences in childhood and adolescence. Sozial Praventivmed 2005;50:281-91.

50. McDougall J, Tsonis M. Quality of life in survivors of childhood cancer: A systematic review of the literature (2001-2008). Support Care Cancer 2009;17:1231-46. Doi: 10.1007/s00520-009-0660-0.

51. Seligman ME, Csikszentmihalyi M. Positive Psychology. An introduction. Am Psychol, 2000;55:5-14.

52. Seligman ME. The past and future of positive psychology. En: Keyes CLM, Haidt J editors. Flourishing: Positive psychology and the life well-lived. Washington, D. C.: American Psychological Association, 2003. p.11-20.

53. Scheier MF, Matthews KA, Owens JF, Magovern GJ Sr, Lefebvre RC, Abbott RA, et al. Dispositional optimism and recovery from coronary artery bypass surgery: The beneficial effects on physical and psychological well-being. J Pers Soc Psychol 1989;57:1024-40.

54. Scheier MF, Carver CS. On the power of positive thinking: the benefits of being optimistic. Curr Dir Psychol Sci 1993;2:2630.

55. Michel G, Taylor N, Absolom K, Eiser C. Benefit finding in survivors of childhood cancer and their parents: further empirical support for the Benefit Finding Scale for Children. Child Care Health Dev 2010;36:123-9. Doi: 10.1111/j.13652214.2009.01034.x.

56. Manzanero AL. Recuerdo de hechos traumáticos: De la introspección al estudio objetivo. Revista de Psicopatología Clínica, Legal y Forense, 2010;10:1-22.

57. Brown R, Kulik J. Flashbulb memories. Cognition 1977; 5:73-99.

58. Pillemer DB. Flashbulb memories of the assassination attempt on President Reagan. Cognition 1984;16:63-80.

59. Schmidt SR. Autobiographical memories for the September 11th attacks: Reconstructive errors and emotional impairment of memory. Mem Cognit, 2004;32:443-54. 